\title{
Correção de contorno gengival pelas técnicas de gengivectomia convencional e minimamente invasiva
}

\author{
Correction of gengival contour for the techniques of conventional and minimally invasive gengivectomy \\ Correo de contorno gengival por las técnicas de gengivectomía convencional y minimamente invasiva \\ Aristony Vinícius Soares CRISTÓVAM ${ }^{1}$ \\ Joana Darc Silva MEDEIROS1 \\ José Henrique de Araújo $\mathbf{C R U Z}^{1}$ \\ Rafaella Mirelly Leite Vasconcelos BRITO² \\ Kadmo Azevedo de FIGUEIREDO ${ }^{3}$ \\ Rachel de Queiroz Ferreira RODRIGUES ${ }^{3}$ \\ João Nilton Lopes de SOUSA \\ ${ }^{1}$ Acadêmico(a) do Curso de Graduação em Odontologia. Centro de Saúde e Tecnologia Rural, Universidade Federal de Campina Grande, \\ UFCG 58708-110 Patos-PB, Brasil \\ ${ }^{2}$ Graduada em Odontologia pela FIP, Faculdade Integrada de Patos, Campus Patos, Patos-PB, Brasil \\ ${ }^{3}$ Professores Doutores da Disciplina de Periodontia do Curso de Graduação em Odontologia. Centro de Saúde e Tecnologia Rural, \\ Universidade Federal de Campina Grande, UFCG 58708-110 Patos-PB, Brasil
}

\begin{abstract}
Resumo
Introdução: A busca por procedimentos cirúrgicos devido às desarmonias dento-gengivais são frequentes. Para um excelente resultado, é necessário um correto diagnóstico, um planejamento adequado, fazendo uso de uma ou mais técnicas melhor indicadas para o caso. Objetivo: O objetivo deste trabalho é relatar um caso clínico de correção do sorriso por meio de cirurgia plástica periodontal. Relato do caso: Participou do estudo a paciente do gênero feminino, com queixas de insatisfação estética no contorno gengival. Após a avaliação clínica e periodontal, observou-se que a paciente apresentava posição labial normal, ausência de perda de inserção, fenótipo periodontal espesso e coroas clínicas dos elementos ântero-superiores desproporcionais. Os elementos 11, 12 e 13 apresentavam coroas clínicas menores que os dentes correspondentes. Optou-se por fazer correção do contorno gengival pela técnica de gengivectomia em bisel externo nos elementos 11 $(1 \mathrm{~mm}), 12(2 \mathrm{~mm})$ e $13(1 \mathrm{~mm})$ com gengivoplastia em todos os dentes do sextante. Resultado: Após reavaliação de 21 dias observou-se que as coroas clínicas dos elementos 12 e 13 apresentavam-se diminuída em decorrência de sua etiologia de erupção passiva alterada. Assim, o procedimento cirúrgico para os elementos 12 e 13 foi uma cirurgia plástica periodontal do tipo gengivectomia e osteotomia minimamente invasiva sem rebatimento de retalho. Conclusão: Após o procedimento cirúrgico e um reparo completo do tecido periodontal, a paciente estava com tamanho das coroas clínicas harmônicas. Portanto, podemos concluir que a utilização de mais de uma técnica de cirurgia plástica periodontal é satisfatória para um resultado final.
\end{abstract}

Descritores: Sorriso; Estética Dentária; Gengivectomia.

\section{Abstract}

Introduction: The search for surgical procedures due to gingival tooth dissonance is frequent. For an excellent result, a proper diagno-sis is required, an adequate planning, making use of one or more techniques best suited for the case. Objective: The objective of this study is to report a clinical case of smile correction through periodontal plastic surgery. Case report: The patient aged 25 years, female, participated in the study, with complaints of aesthetic dissatisfaction in the gingival contour. After clinical and periodontal evaluation, the patient had normal labial position, absence of insertion loss, thick periodontal phenotype and clinical crowns of the disproportionate anterosuperior elements. Elements 11, 12 and 13 presented smaller clinical crowns than the corresponding teeth. We chose to correct the gingival contour by means of external bevel gingivectomy in the $11(1 \mathrm{~mm}), 12(2 \mathrm{~mm})$ and $13(1 \mathrm{~mm})$ elements with gingivoplasty on all teeth of the sextant. Results: After a reassessment of 21 days, it was observed that the clinical crowns of elements 12 and 13 were decreased due to their etiology of altered passive eruption. Thus, the surgical procedure for elements 12 and 13 was a periodontal plastic surgery of the gingivectomy type and minimally invasive osteotomy without flap resting. Conclusion: After the surgical procedure and a complete repair of the periodontal tissue, the patient had clinical crown size harmonics. Therefore, we can conclude that the use of more than one periodontal plastic surgery technique is satisfactory for a final result.

Descriptors: Smiling; Esthetic, Dental; Gingivectomy.

\section{Resumen}

Introducción: La búsqueda de procedimientos quirúrgicos debido a las desarmonías de las encías es frecuente. Para un excelente resultado, es necesario un correcto diagnóstico, una planificación adecuada, haciendo uso de una o más técnicas mejor indicadas para el caso. Objetivo: El objetivo de este trabajo es relatar un caso clínico de corrección de la sonrisa por medio de cirugía plástica periodontal. Relato del caso: Participó del estudio paciente del sexo femenino, con quejas de insatisfacción estética en el contorno gingival. Después de la evaluación clínica y periodontal, se observó que la paciente presentaba posición labial normal, ausencia de pérdida de inserción, fenotipo periodontal espeso y coronas clínicas de los elementos antero-superiores desproporcionados. Los elementos 11, 12 y 13 presentaban coronas clínicas menores que los dientes correspondientes. Se optó por hacer corrección del contorno gingival por la técnica de gingivectomía en bisel externo en los elementos $11(1 \mathrm{~mm}), 12(2 \mathrm{~mm})$ y $13(1 \mathrm{~mm})$ con gingoplastia en todos los dientes del sextante. Resultado: Después de la reevaluación de 21 días se observó que las coronas clínicas de los elementos 12 y 13 se presentaban disminuidas como consecuencia de su etiología de erupción pasiva alterada. Así, el procedimiento quirúrgico para los elementos 12 y 13 fue una cirugía plástica periodontal del tipo gingivectomía y osteotomía mínimamente invasiva sin rebatimiento al por menor. Conclusión: Después del procedimiento quirúrgico y una reparación completa del tejido periodontal, la paciente tenía el tamaño de las coronas clínicas armónicas. Por lo tanto, podemos concluir que la utilización de más de una técnica de cirugía plástica periodontal es satisfactoria para un resultado final.

Descriptores: Sonrisa; Estética Dental; Gingivectomía.

\section{INTRODUÇÃO}

O ambiente social em que estamos, tem- se mostrado rigoroso no que tange a estética bucal, devido a isso, ocorre um aumento na procura por procedimentos odontológicos que favoreçam um sorriso harmônico ${ }^{1}$. Observa-se um número maior de pacientes que procuram procedimentos estéticos, levando os cirurgiões dentistas a melhorarem as técnicas utilizadas para esses fins ${ }^{2}$.

A estética do sorriso promove expressões faciais diferentes, sendo o tecido gengival de real importância nesta avaliação ${ }^{3}$. Vários são os fatores que podem interferir numa estética satisfatória de um 
sorriso considerado não gengival. Entre eles podemos citar: erupção dentária passiva alterada, coroa clínica dental diminuída, crescimento exagerado da maxila no sentido vertical e diminuição da espessura do lábio superior ${ }^{4}$.

Tanto os especialistas quantos os pacientes, relatam que a estética do sorriso é prejudicada quando existe uma exposição gengival excessiva ao sorrir, que está associada à posição do lábio superior em relação à margem gengival, sendo influenciada pelo sexo e a idade dos indivíduos ${ }^{5}$. A posição anatômica dentária em relação com a gengiva, o lábio e o padrão facial de cada paciente, sugere um formato e contorno dos dentes, específicos para uma melhor estética da linha do sorriso ${ }^{6}$.

Diante de tantos fatores que influenciam na estética do sorriso, é imprescindível um correto planejamento da linha do sorriso para resolução do sorriso gengival. A técnica de gengivectomia convencional e minimamente invasiva tem sido amplamente descrita por seus resultados satisfatórios ${ }^{7}$.

O objetivo deste estudo foi relatar um caso clínico de correção de contorno gengival pela técnica gengivectomia convencional e osteotomia sem deslocamento de retalho.

\section{CASO CLÍNICO}

\section{- Exame clínico e periodontal}

Este estudo tratou-se de um relato de um caso clínico de uma paciente, 25 anos, gênero feminino, leucoderma, sem alterações sistêmicas e com saúde periodontal e ausência de hiperplasia gengival, que procurou o Projeto de Extensão em Periodontia Clínica e Cirúrgica (PROEPECC) da Universidade Federal de Campina Grande - UFCG/CSTR com queixas de insatisfação estética no contorno gengival. Após a avaliação clínica e periodontal, observou-se que a paciente apresentava posição labial normal, ausência de perda de inserção, fenótipo periodontal espesso com larga faixa de mucosa queratinizada, linha cervical assimétrica (linha dos zênites gengivais) e coroas clínicas dos elementos anterossuperiores desproporcionais ao comparar com os dentes homólogos. Os elementos 11, 12 e 13 apresentavam coras clínicas menores que os dentes correspondentes do lado esquerdo (Figuras 1 e 2). Foi realizado o exame radiográfico, constatando que não havia perda óssea ou qualquer alteração no periodonto de sustentação e exibindo dentes hígidos, sem tratamento endodôntico e restaurador (Figura 3).

Durante exame periodontal detalhado, foram avaliados os seguintes parâmetros clínicos: profundidade de sondagem, recessão gengival, mucosa ceratinizada e gengiva inserida. Após estas avaliações, constatou-se profundidades de sondagem inferior a $3 \mathrm{~mm}$, ausência de sangramento a sondagem e de recessões, caracterizando a inexistência de perda de inserção clínica, conforme observado no Quadro 1.

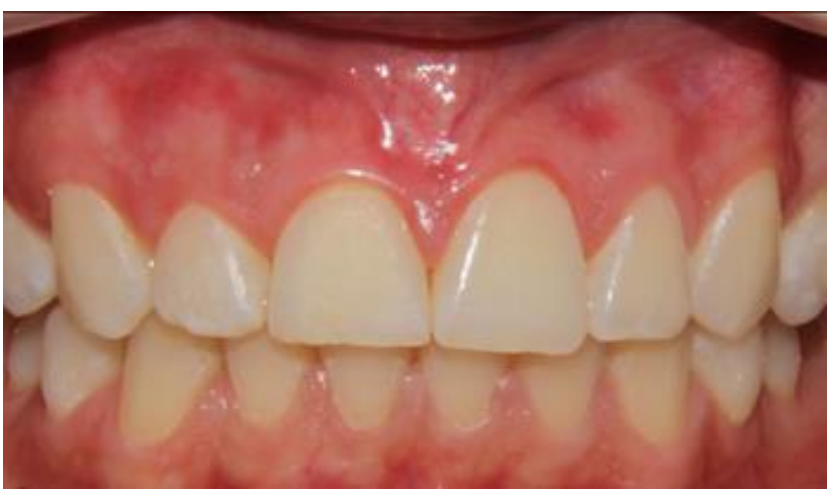

Figura 1: Observa-se a imagem dos dentes em máxima intercuspidação habitual (MIH) e a estética do contorno gengival.

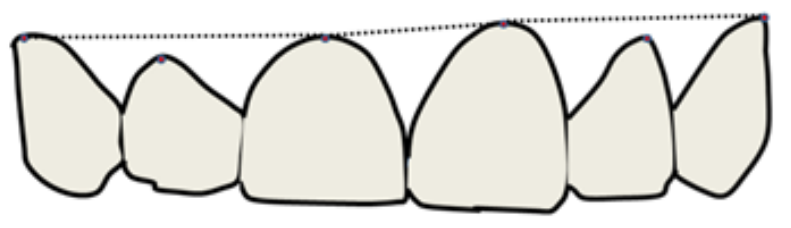

Figura 2: Formato e tamanho das coroas clínicas dos elementos anterossuperiores e a linha dos zênites gengivais.

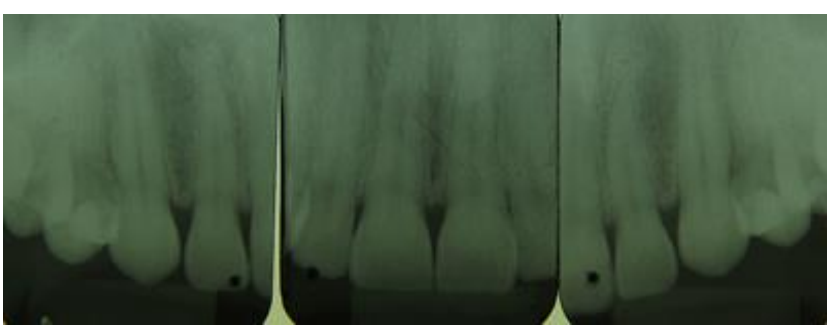

Figura 3: Radiografias periapicais dos elementos 13 ao 23.

Quadro I. Exame periodontal detalhado (periograma)

\begin{tabular}{|l|l|l|l|l|l|l|l|l|l|}
\hline & $\begin{array}{l}\text { COROA } \\
\text { CLÍNICA }\end{array}$ & R & S & R & S & R & S & mm & Mm \\
\hline $\mathbf{1 3}$ & $8 \mathrm{~mm}$ & 0 & 2 & 0 & 2 & 0 & 2 & 9 & 6 \\
\hline $\mathbf{1 2}$ & $5.5 \mathrm{~mm}$ & 0 & 2 & 0 & 2 & 0 & 2 & 9 & 6 \\
\hline $\mathbf{1 1}$ & $7 \mathrm{~mm}$ & 0 & 2 & 0 & 2,5 & 0 & 3 & 7 & 5 \\
\hline $\mathbf{2 1}$ & $8 \mathrm{~mm}$ & 0 & 2 & 0 & 1.0 & 0 & 2 & 8 & 5 \\
\hline $\mathbf{2 2}$ & $7,5 \mathrm{~mm}$ & 0 & 2 & 0 & 2 & 0 & 2 & 9 & 6 \\
\hline $\mathbf{2 3}$ & $9 \mathrm{~mm}$ & 0 & 2 & 0 & 0,5 & 0 & 3 & 10 & 4 \\
\hline
\end{tabular}

$\mathrm{R}$ - recessão gengival; $\mathrm{S}$ - profundidade de sondagem; $\mathrm{MV}$ - mesio vestibular; $\mathrm{V}$ - vestibular DV disto vestibular; MC - mucosa ceratiniza; GI - gengiva inserida

Este caso clínico foi submetido ao Comitê de Ética em Pesquisa das Faculdades Integradas de Patos-PB (CAAE: 59592016.8.0000.5181). O plano de tratamento foi explicado para a paciente, a qual assinou o Termo de Consentimento Livre Esclarecido (TCLE), autorizando a realização do procedimento cirúrgico e publicação deste caso clínico.

- Planejamneto cirúrgico

Como se observou uma maior profundidade de sondagem no lado direito do sextante 2, optou-se 
inicialmente por fazer a correção do contorno gengival pela técnica de gengivectomia em bisel externo nos elementos $11(1 \mathrm{~mm}), 12(2 \mathrm{~mm})$ e 13 (1 $\mathrm{mm})$ com gengivoplastia em todos os dentes do sextante (Figura 4). Na reavaliação de 21 dias, observou-se que o elemento 11 apresentava um contorno gengival compatível com o 21 , porém os elementos 12 e 13 ainda apresentavam coras clínicas menores que seus homólogos do lado esquerdo, persistindo a alteração do contorno gengival nos dentes 12 e 13 (Figura 5). Esta alteração no contorno gengival destes elementos estava relacionada à erupção passiva alterada, havendo a necessidade de osteotomia para posicionar a margem gengival mais apicalmente e aumentar suas coroas clínicas.

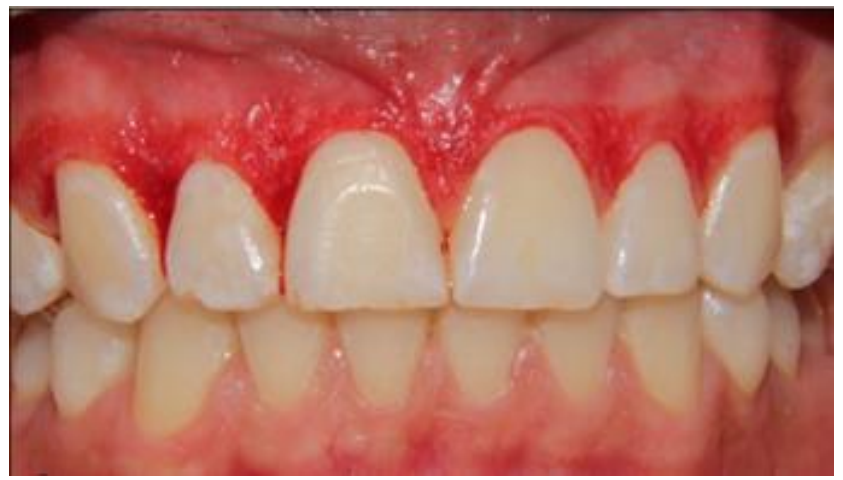

Figura 4: Aspecto final da gengivectomia e gengivoplastia.

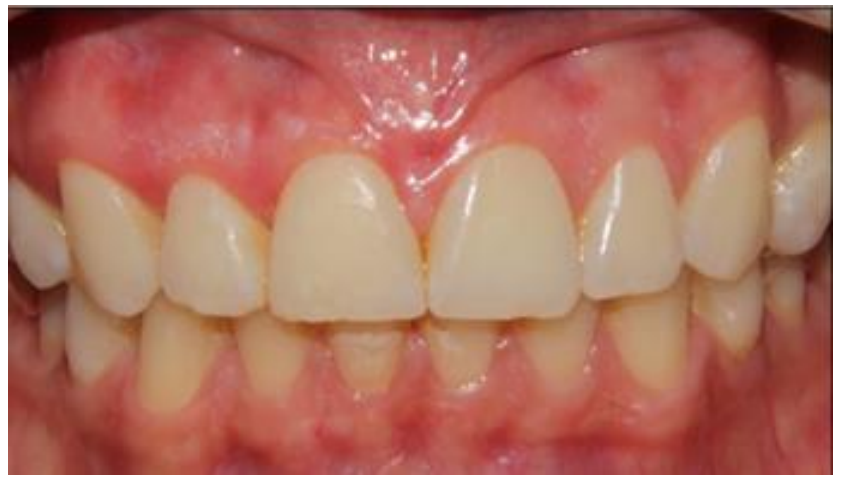

Figura 5: Pós-operatório de 21 dias.

O procedimento cirúrgico escolhido para correção da erupção passiva alterada nos elementos 12 e 13 foi uma cirurgia plástica periodontal do tipo gengivectomia e osteotomia suficientemente invasiva sem rebatimento de retalho.

No procedimento cirúrgico, realizou-se antissepsia extra e intrabucal com digluconato de clorexidina 2\% (Dentscare LTDA, Joinville-SC, Brasil) e 0,12\% (Rioquímica LTDA, São José do Rio Preto-SP, Brasil), respectivamente. Em seguida, anestesia com Articaína 2\% com vasoconstritor (DFL Indústria e Comércio S.A., Rio de Janeiro-RJ, Brasil), utilizando a técnica infiltrativa no fundo de sulco vestibular e nas papilas vestibulares e palatinas.

Com uma sonda periodontal do tipo Williams (Golgran, São Caetano do Sul-SP, Brasil), foi realizada a marcação, por meio de sondagem transgengival, da faixa de gengiva a ser removida na gengivectomia de um $1 \mathrm{~mm}$ nos dentes 12 e 13 (Figuras 6 e 7). A partir de cada ponto marcado, na altura do futuro zênite gengival, foi planejado o contorno da linha de incisão com a ponta da lâmina de bisturi $15 \mathrm{C}$, penetrando superficialmente no tecido gengival e formando uma linha fina visualizada devido ao sangramento (Figuras 8 e 9).

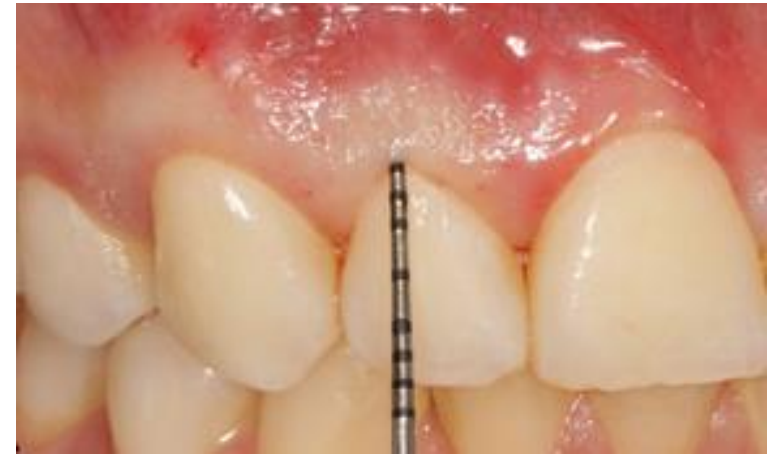

Figura 6: Planejamento da gengivectomia nos elementos 12 e 13

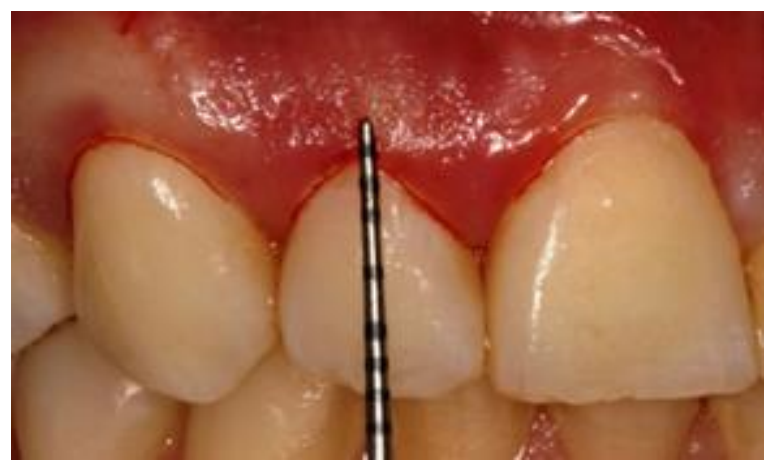

Figura 7: Planejamento da gengivectomia nos elementos 12 e 13: profundidade de sondagem.

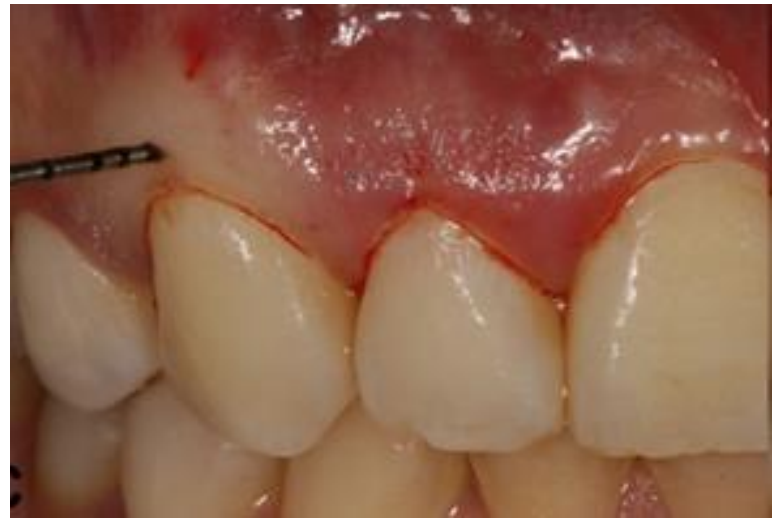

Figura 8: Marcação do zênite gengival cirúrgico.

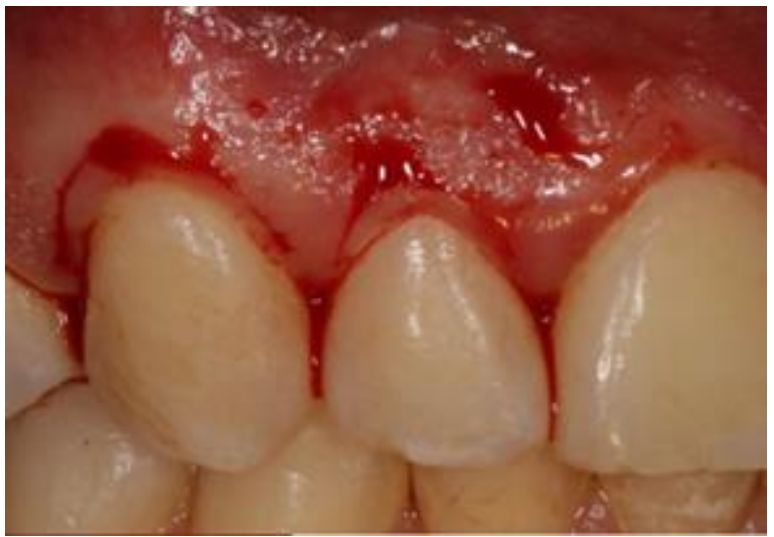

Figura 9: Sondagem transgengival. 
Para um contorno adequado, alinhou-se o zênite do canino para distal. As incisões para remoção do excesso gengival foram realizadas com uma lâmina de bisturi 15C (Lamedid Comercial e Serviços LTDA, Barueri-SP, Brasil) posicionada a $90^{\circ} \mathrm{em}$ relação ao tecido periodontal. Imediatamente após as incisões, o tecido gengival foi removido com auxílio de cureta periodontal de Gracey 5-6 (Golgran, São Caetano do Sul-SP, Brasil). A margem gengival cirúrgica ficou posicionada ao nível a junção cemento-esmalte (Figura 10).

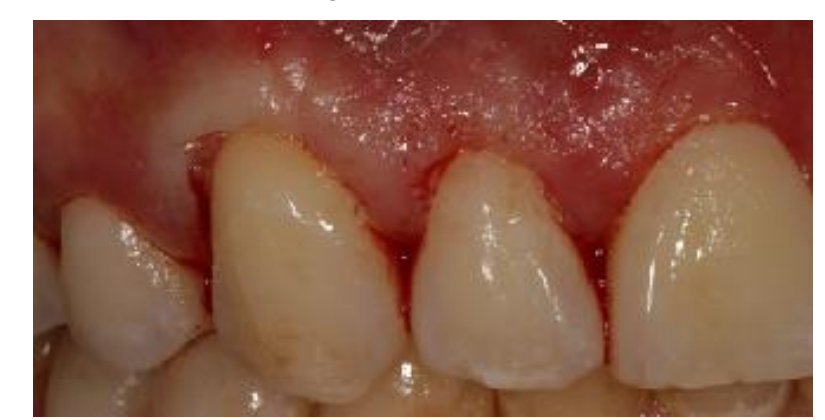

Figura 10: Coroas clínicas e contorno gengival cirúrgico imediatamente após a gengivectomia.

Ao remover o tecido gengival incisado, uma sonda periodontal do tipo HWO OMS (Golgran, São Caetano do Sul-SP, Brasil) foi utilizada para avaliar a distância da crista óssea alveolar (COA) à junção cemento esmalte (JCE), que foi $0 \mathrm{~mm}$ nos dois elementos, confirmando clinicamente a erupção passiva alterada (Figura 11).

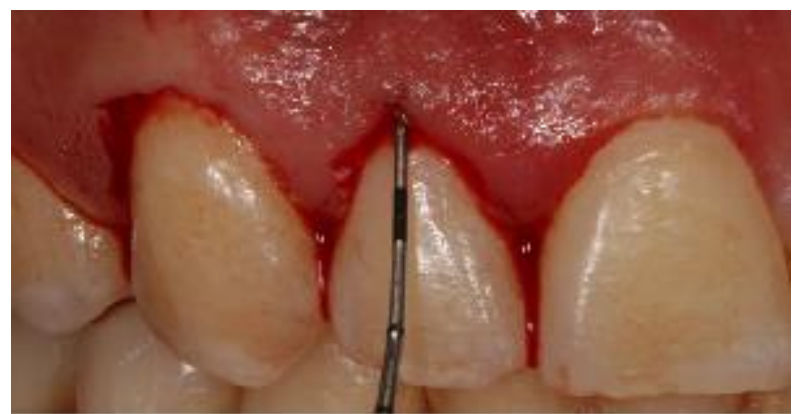

Figura 11: Avaliação da posição da COA em relação à JCE.

Em seguida foi realizada osteotomia com Cinzel Mini-Ochsenbein $\mathrm{N}^{\circ} 2$. Sua lamina ativa foi posicionada a uma angulação de aproximadamente $5^{\circ}$ em relação ao longo eixo do dente (Figuras 12, 13 e 14).

Movimentos leves apicais e de rotação foram realizados no cinzel para remover a crista óssea alveolar, distanciando-a em $2 \mathrm{~mm}$ a da margem gengival cirúrgica, aumentando as coroas clínicas dos elementos e restabelecendo as distâncias biológicas periodontais. Após esta etapa, realizou-se remoção dos fragmentos ósseos, raspagem e alisamento em 1 $\mathrm{mm}$ subgengival da superfície radicular e irrigação copiosa com soro fisiológico (Figuras 15 e 16).

Após a osteotomia e osteoplastia, foi realizada irrigação abundante com solução salina a 0,9 \% (Eurofarma Laboratórios LTDA, São Paulo-
SP, Brasil) e não realizou sutura (Figura 17). Em seguida, a paciente foi orientada com relação às instruções de higiene oral e cuidados pós-operatórios. Foi prescrito Dipirona $500 \mathrm{mg}$ de 6/6horas por 48 horas apenas em caso de dor.

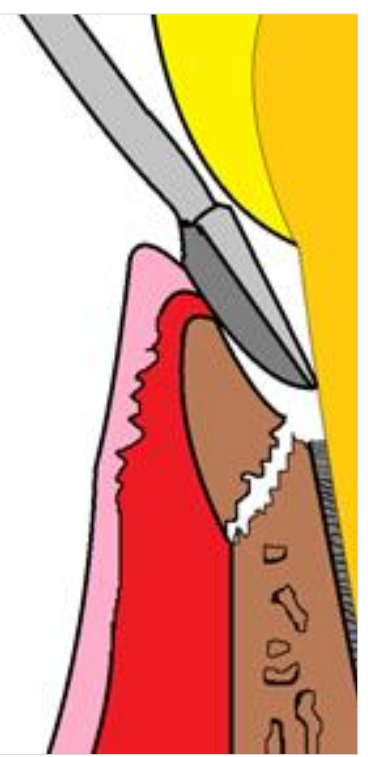

Figura 12: Ilustração da posição do cinzel MiniOchsenbein $\quad \mathrm{N}^{\circ} 2$. e da osteotomia

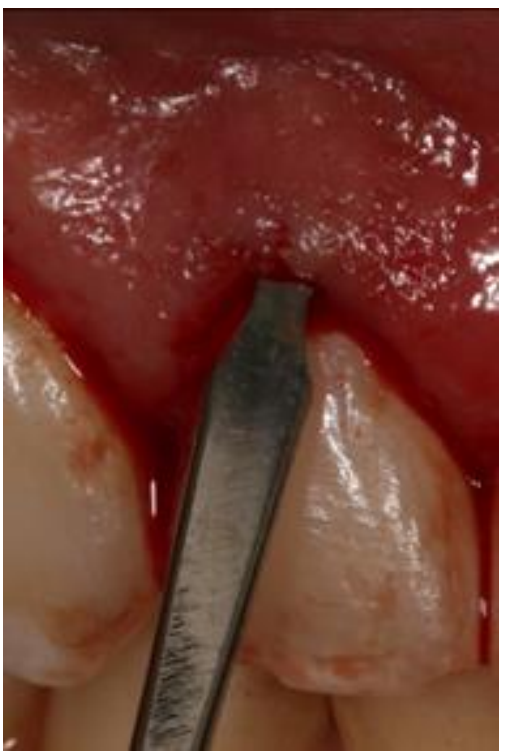

\section{Figura 13:}

Posicionamento do Cinzel Mini-

Ochsenbein $\mathrm{N}^{\circ} 2$ (visão frontal)

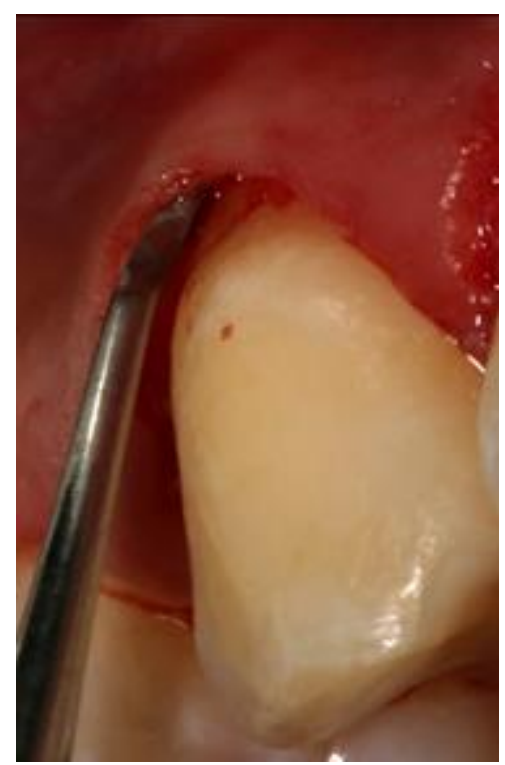

Figura 14:

Posicionamento do

Cinzel Mini-

Ochsenbein $\mathrm{N}^{\circ} 2$

(visão intrassucular) 


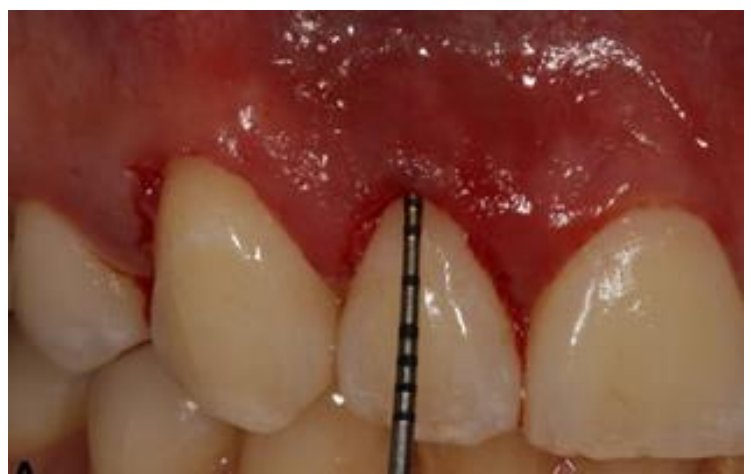

Figura 15: Avaliação da profundidade da osteotomia (2 mm).

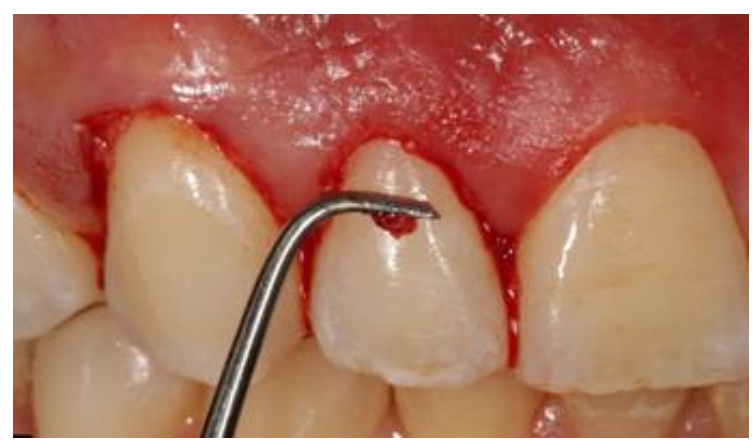

Figura 16: Remoção dos fragmentos ósseos e RAR em $1 \mathrm{~mm}$ subgengival.

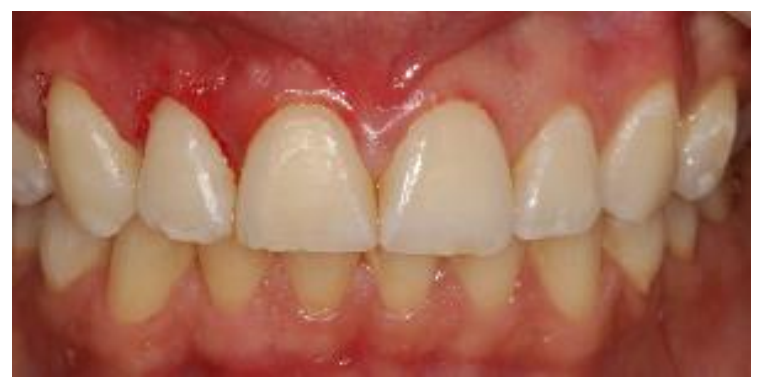

Figura 17: Pós-operatório imediato.

No pós-operatório de 26 dias, a paciente apresentava um quadro de saúde periodontal, ausência de sangramento gengival, contornos gengivais simétricos, com aspecto de casca de laranja (Figuras 18 e 19). A paciente relatou satisfação com o resultado.

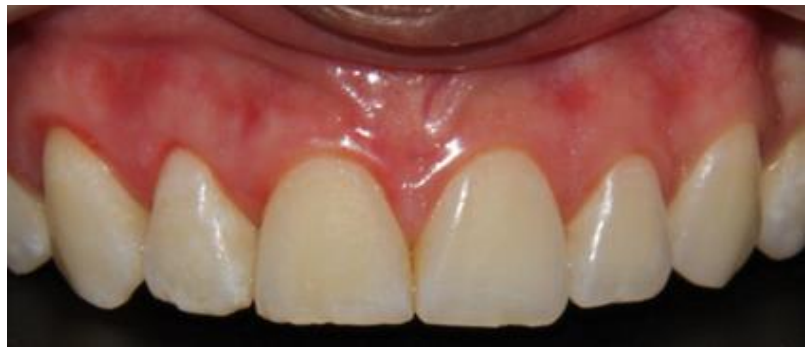

Figura 18: Pós-operatório de 26 dias.

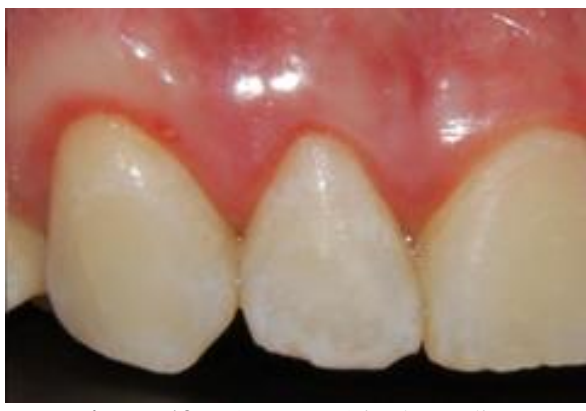

Figura 19: Pós-operatório de 26 dias.
No pós-operatório de 9 meses, observou-se que a paciente apresentou um sorriso mais agradável e harmônico (Figura 20) e a mesma relatou melhora de sua autoestima.

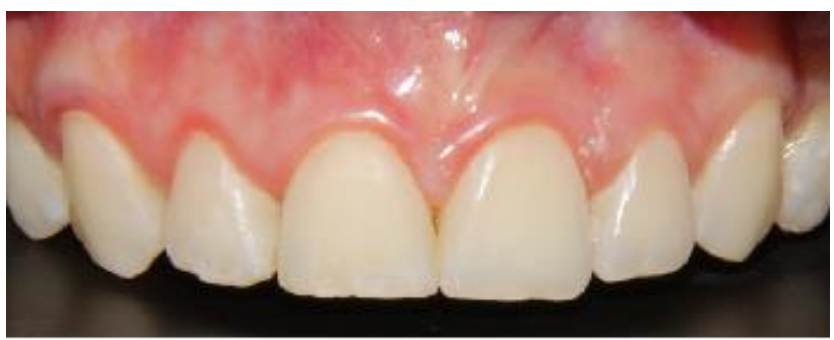

Figura 20: Pós-operatório de 9 meses.

DISCUSSÃO

A estética periodontal ou estética vermelha tem sido muito discutida e valorizada em busca da harmonia do $\operatorname{sorriso}^{8}$, fazendo com que um maior número de pacientes procure procedimentos estéticos, levando os cirurgiões-dentistas a melhorarem as técnicas utilizadas para esses fins ${ }^{2}$. A erupção passiva alterada é uma condição que interfere na estética do sorriso, por originar coroas clínicas curtas e estar associada ao sorriso gengival $^{9-12}$ ou ao contorno inadequado ${ }^{13}$. Neste caso clínico, a paciente procurou o tratamento periodontal para corrigir alteração no contorno gengival que lhe causava desconforto ao sorrir, corroborando com os estudos citados acima.

Em situações de erupção passiva alterada, ou seja, existência de grande exposição de gengiva acompanhada de coroas clínicas curtas, há indicação precisa para a execução de aumento de coroa clínica: tratamento do sorriso gengival através de cirurgia periodontal ${ }^{8}$. Assim como relatado por Faria et al. ${ }^{11}$, a paciente deste estudo foi diagnosticada com erupção passiva alterada nos elementos 12 e 13 . Avaliaram-se a exposição dentária durante o repouso, a saúde do periodonto, a musculatura do lábio superior bem como sua dimensão e do tamanho das coroas clínicas de incisivo ao canino superior nos dois lados da arcada.

O tratamento de escolha para corrigir as alterações estéticas do sorriso, depende da gravidade do caso ${ }^{9,11}$. A dificuldade de diagnóstico deste caso ocorreu devido ao fato da erupção passiva estar relacionada a dentes isolados. Por isso, realizou-se inicialmente gengivectomia/gengivoplastia para corrigir a alteração contorno gengival, porém as coroas clínicas dos elementos 12 e 23, que apresentavam erupção passiva alterada, não foram aumentadas como planejado. Assim, não foi possível corrigir o defeito estético por esta técnica, havendo a necessidade de se realizar osteotomia nestes elementos, para restabelecer o espaço adequado e para acomodar as estruturas periodontais quem fazem parte do espaço biológico ${ }^{14}$.

O correto diagnóstico da causa e a adequada 
seleção da técnica cirúrgica para a correção do sorriso gengival são de fundamental importância para o sucesso do tratamento, sendo este previsível e satisfatório $^{11}$. Na correção de erupção passiva alterada relacionada à estética do sorriso, o periodontista pode optar por duas técnicas para restabelecer as distâncias biológicas e aumentar as coroas clínicas dos elementos: gengivectomia e osteotomia com cinzeis e brocas após deslocamento total do retalho mucogengival ${ }^{15,16}$ ou gengivectomia e osteotomia sem deslocamento de retalho, também denominada de minimamente traumática $^{8}$ ou minimamente invasiva ${ }^{13}$.

Em ambas as técnicas, as distâncias biológicas são estabelecidas distanciando a crista óssea alveolar em $3 \mathrm{~mm}$ da junção cementoesmalte por meio de osteotomia ${ }^{15}$. No presente caso clínico, a osteotomia foi realizada em $2 \mathrm{~mm}$ pela técnica minimamente traumática, sem deslocamento de retalho, com o cinzel Mini-Ochsenbein $\mathrm{N}^{\circ} 2$ que apresenta a lâmina ativa pequena e delicada, o que favoreceu ressecção óssea sem danos a mucosa. Nesta técnica é fundamental a utilização de microcinzeis, pois a remoção óssea é via sulco gengival $^{14}$. Esta técnica foi escolhida, pois se verificou, durante na sondagem, que a paciente apresentava uma espessura de tecido ósseo vestibular possível de ser removida via sulco, sem necessidade de retalho aberto. Para Pontes et al. ${ }^{13}$, quando se tratar de um caso de fenótipo ósseo espesso e/ou exostoses ósseas, será necessário utilizar a técnica convencional, com retalho aberto, para exposição do tecido ósseo e osteoplastia, com a remoção de osso não apenas no sentido coronário-apical, como também em espessura na região vestibular.

A escolha de uma determinada técnica para aumento de coroa clínica é dependente de inúmeros fatores, incluindo a quantidade de tecido gengival queratinizado e ósseo remanescente ${ }^{13}$. Neste caso, a incisão marginal e a remoção do "colar" gengival estavam indicadas, pois a paciente apresentava uma adequada faixa de tecido gengival queratinizado.

A técnica de gengivectomia com osteotomia sem deslocar retalho pode ser indicada em vários casos de correção da estética do sorriso ${ }^{8}$. Apesar desta técnica não necessitar de sutura, o sangramento pós-operatório não foi significativo e a paciente relatou que em poucas horas foi cessado totalmente. Nas avaliações de controle, observaram-se uma boa recuperação do tecido periodontal, com o mínimo desconforto pós-operatório para o paciente e sem necessidade de consumo de analgésico para sintomas de dor. Estes resultados podem ser em virtude do procedimento não causar prejuízos ao suprimento sanguíneo da região operada; não danificar os tecidos moles, minimizando assim a morbidade trans e pósoperatória; não necessitar de elevação de retalho e de suturas $^{17,10}$.
No resultado final do caso, observaram-se a correção do contorno gengival, harmonização dentogengival e melhora da auto estima da paciente, verificando que a técnica de osteotomia minimamente invasiva mostrou-se eficaz. Resultado semelhante ao encontrado por Trevisani e Meuseu ${ }^{8}$. Em geral, os resultados demonstraram que esta técnica minimamente invasiva proporciona sucesso em relação ao aumento das coroas clínicas dos elementos anteriores superiores, melhora na harmonia do sorriso, além de satisfação estética ${ }^{14}$. Corroborando com Alvarenga et al. ${ }^{17}$, a paciente deste estudo também relatou melhora na autoestima após a correção cirúrgica da estética vermelha do sorriso.

\section{CONSIDERAÇÕES FINAIS}

A técnica de gengivectomia e osteotomia minimamente invasiva pode ser uma excelente opção para tratar os casos de alterações estéticas em virtude da exposição gengival excessiva ao sorrir e o inadequado contorno da margem gengival, restabelecendo a harmonia do sorriso.

\section{REFERÊNCIAS}

1. Souza PC. Garzon AC da M, Sampaio JEC. Estética Periodontal: Relato de um caso. Rev Bras Cir Periodontia. 2003;1(4):262-67.

2. Bertholdo G, Silveira I, Caporossi L, Albino LGB, Monsano R. Estética integrada: Reestabelecendo a harmonia do sorriso. Prosthes Lab Sci 2015;4(16):341-44.

3. Dutra MB, Ritter DE, Borgatto A, Derech CDA, Rocha R. Influência da exposição gengival na estética do sorriso. Dental Press J Orthod. 2011; 16(5):111-18.

4. Sousa SJB, Magalhães D, Silva GR, Soares CJ, Soares PFB, Santos Filho PCF. Cirurgia plástica periodontal para correção de sorriso gengival associado à restaurações em resina composta: Relato de caso clínico. Rev Odontol Bras Central. 2010;19(51):362-66.

5. Seixas MR, Costa-Pinto RA, Araújo TM. Checklist dos aspectos a serem considerados no diagnóstico e tratamento do sorriso gengival. Dental Press J Orthod. 2011;16(2):131-57.

6. Pedron IG, Utime ER, Tancredi ARC, Perrela A, Perez FEG. Sorriso gengival: cirurgia ressectiva coadjuvante à estética dental. Odonto 2010;18(35):87-95.

7. Pires CV, Sousa CGLG, Menezes SAF. Procedimentos plásticos periodontais em paciente com sorriso gengival - relato de caso. R Periodontia. 2010;20(1):48-53.

8. Trevisani RS, Von Meusel DRDZ. Aumento de coroa clínica em dentes anteriores - relato de caso clínico. J Oral Invest. 2014;3(2):19-24.

9. Bertolini PFR, Biondi Filho O, Kiyan VH, Saraceni CHC. Recuperação da estética do 
sorriso: cirurgia plástica periodontal e reabilitação protética. Rev Ciênc Méd. 2011;20(5-6):137-43.

10.Carvalho PA, Alfaya TA, Costa RC, Bussadori SK, Vieira EO, Gouvêa CVD. Correção de erupção passiva alterada por meio de cirurgia plástica periodontal. PerioNews. 2013;7(5):505-9.

11. Braga MS, Nascimento LMQ, Camargo EB, Veloso Filho JMSC, Falcaõ EP, Zuza EP et al.Cirurgia plástica para correção de erupção passiva alterada. Braz J Periodontol. 2015;24(4):64-8.

12.Faria GJ, Barra SG, Vieira TR, Oliveira PAD. A importância do planejamento multidisciplimar para correção do sorriso gengival: Relato de caso. Rev Fac Odontol Lins. 2015;25(1):61-5.

13.Pontes A, Duarte PM, Oliveira ACG, Coelho EF, Esteves FM, Mello GBR et al. Aumento de coroa clínica estético minimamente invasivo: relato de caso de 12 meses. Rev Saúde. 2016;10(3-4):55-64.

14.Fernandes TV, Chaves EAL, Souza Jr EJ. Integração periodontia/dentística na restauração estética anterior.Fulldent.Sci. 2014;6(21):7-15.

15.Clozza E, Suzuki T, Mohajer KA. Tratamento de erupção passiva alterada para melhorar a estética do sorriso. Dicas de Perodontia.2014;3(1):36-41.

16. Alvarengua DV, Santana CLV, Oliveira FRDTS, Rodrigues RQF, Ribeiro RA, Souza JNL. Interrelação na periodontia/dentística na correção de sorriso gengival: relato de caso clínico. Periodontia. 2018;28(2):53-9.

17.Lobo M. Aumento de coroa anterior estético minimamente traumático: caso clínico selecionado. Brasil Dentistry Clínica. 2011:19-26.

\section{CONFLITO DE INTERESSES}

Os autores declaram não haver conflitos de interesse.

\section{AUTOR PARA CORRESPONDENCIA}

\section{José Henrique de Araújo Cruz}

henrique_araujo1992@ hotmail.com

Submetido em 04/1 1/2018

Aceito em 12/03/2019 